\title{
A multi-state model for kidney disease progression
}

\author{
M.K. Lintu ${ }^{a}$, K.M. Shreyas ${ }^{\mathrm{b}}$, Asha Kamath ${ }^{\mathrm{a},{ }^{*}}$ \\ ${ }^{a}$ Department of Data Science, Manipal Academy of Higher Education, Manipal, 576104, Karnataka, India \\ ${ }^{\mathrm{b}}$ Department of Pharmacy Practice, Manipal Academy of Higher Education, Manipal, 576104, Karnataka, India
}

\section{A R T I C L E I N F O}

\section{Keywords:}

Multi-state model

Kidney disease

Transition intensity

Intermediate events

Disease progression

\begin{abstract}
A B S T R A C T
Background: Understanding the progression of kidney disease is of great interest among clinicians. The multi-state model is an adequate tool to model the effects of covariates that influence the onset, progression, and regression of kidney function.

Objective: The goal of the present study is to propose a stochastic model for kidney disease progression and to demonstrate the application of the same.

Methodology: We proposed a semi-parametric continuous time homogeneous multi-state Markov model for the kidney disease progression data obtained from a retrospective study of 225 patients prescribed with colistin (a reemerging antibiotic) in a tertiary care hospital in coastal Karnataka. Different stages of kidney disease were defined based on the Kidney Disease Improving Global Outcome (KDIGO) score. The model consists of three transient states, and an absorbing state death. Covariate effects on the bidirectional transition rates were estimated using the multi-state model.

Results: We used the data of 225 patients to see their kidney disease progression. All the patients were under colistin therapy. The median length of hospital stay was 21 days. A total of $83(36.89 \%)$ patients died in the hospital. The prognostic factors such as gender, hypertension, sepsis, and surgery are significant factors affecting kidney disease in different stages.

Conclusion: The findings of the study will be useful for public health policymakers to implement the policies and treatment plans to improve the survival of the patients. Moreover, modelling the disease progression helps in understanding the expected burden of the disease.
\end{abstract}

\section{Introduction}

Kidney disease is an important public health problem. However, early intervention can avoid kidney problems permanently. The adverse outcomes and progression of chronic disease such as kidney disease can be controlled by adequate medications and lifestyle changes. As kidney diseases often end up with hospitalization, modelling the length of stay, survival, and progression of kidney disease is of great interest among clinicians.

Standard survival approaches such as the Kaplan-Meier method or Cox proportional hazards model are sufficient to handle the simple survival settings with no intermediate events. However, the kidney disease progression is an example of a complex process with different intermediate events. The multi-state model is an efficient way to handle complex processes such as this. The subjects can be at one state at the beginning of the study, further pass through different states and eventually end up in a final state. These transitions of a subject can be modeled and the risk factors associated with survival times of different transitions can be identified using a multi-state model. The model estimates the effect of different clinical and demographic factors on complex disease processes. ${ }^{1-3}$

The multi-state model is commonly used in cancer studies as there are different stages of cancer indicating disease progression. ${ }^{4,5}$ Similarly, there are studies conducted to identify the risk factors influencing AIDS progression in HIV patients. ${ }^{6-8}$ Diseases such as diabetes, chronic kidney disease, etc. can also be efficiently handled with a multi-state model rather than a simple survival model. ${ }^{9,10}$

In this article, we proposed a multi-state model for the kidney disease progression of the patients receiving colistin during their hospital stay. Case records of 225 patients were retrieved and their KDIGO scores at different time points were accessed for a clinical companion paper. Length of hospital stay is the number of days from admission till their death/discharge. During the hospital stay, the patients were passing through different stages of disease severity. We defined the process with

\footnotetext{
* Corresponding author.

E-mail address: asha.kamath@manipal.edu (A. Kamath).
} 
Table 1

Different stages of kidney disease and states derived from it.

\begin{tabular}{ccc}
\hline Stage & KDIGO score & State \\
\hline 1 & $\geq 90$ \\
2 & $60-89$ & - 1 (Mild) \\
3 & $30-59$ & 2 (Moderate) \\
4 & $15-29$ \\
5 & $<15$ &
\end{tabular}

KDIGO: Kidney Disease Improving Global Outcomes

Table 2

Sample data layout for a multi-state model.

\begin{tabular}{llllll}
\hline Patient ID & No. of days & State & Age & Gender & Pneumonia \\
\hline 2898554 & 5 & 2 & 36 & 1 & 0 \\
2898554 & 17 & 3 & 36 & 1 & 0 \\
2898554 & 19 & 2 & 36 & 1 & 0 \\
2898554 & 20 & 1 & 36 & 1 & 0 \\
2836110 & 160 & 2 & 58 & 0 & 1 \\
2836110 & 162 & 4 & 58 & 0 & 1 \\
\hline
\end{tabular}

four states. Three transient states derived from the KDIGO score are given in Table 1.

From different epidemiological studies, it is clear that different biomarkers and comorbidities play important role in the progression of kidney disease. We estimated the effects of covariates that influence the onset, progression, and regression of renal function using the multi-state model. We also provide a demonstration for data documentation, analysis, and interpretation so that readers can implement the multistate model easily. To the best of our knowledge, the multi-state Markov model has not been used to model colistin-induced kidney disease progression.

In the rest of the paper, we present the data, explain the methodology, and discuss the results.

\section{Materials and methods}

\subsection{Data description}

The retrospective study was originally conducted to understand the risk factors associated with colistin-induced nephrotoxicity in patients treated at the Department of Medicine, Kasturba Hospital, Manipal, from January 2016 to December 2017. We further investigate the inhospital mortality, length of hospital stay, and kidney disease progression. Out of the total 600 case records screened, 225 patients were appropriate for the present study. Hospital case records of these 225 patients form the basis of the present study.

KDIGO score was accessed at different time points to monitor the kidney function improvement and decline. The prognostic factors such as age, gender, diabetes, hypertension, etc. were recorded for each patient. Similar studies in the literature approached the problem with logistic regression or ordinary survival analysis by considering the endpoint as renal function decline. These methods neglect the progression and regression transitions across the different states. The multi-state model provides an in-depth understanding of the general renal function improvement or deterioration patterns and death outcome in terms of transition probabilities. A transition is a change of state over the course of a study and transition probability is the probability of moving from

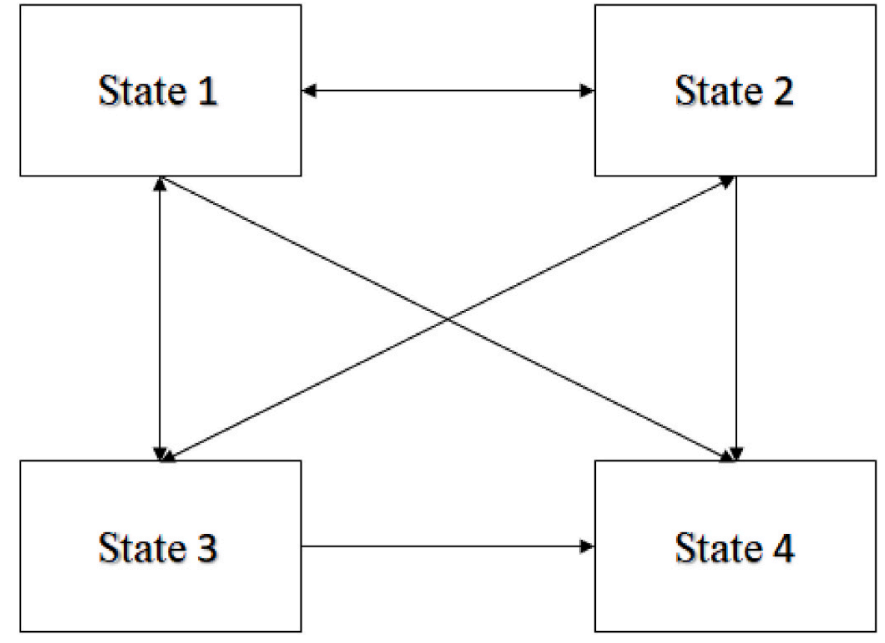

Fig. 1. The proposed 4-state model.

one state to another. Most of the patients seemed to oscillate between state 1 , state 2 , and state 3 multiple times, which indicates that these states are reversible. Transition is reversible when the subject moves back and forth between two states.

Data arrangement is an important step in fitting a multi-state model. The unique ID given to each patient takes care of the grouping of a single subject. Hence, care should be given while documenting the data to make sure that all the information from a single patient has been covered under the same unique ID. The sample data layout is given in Table 2 .

\subsection{Multi-state model}

The kidney disease progression can be visualized as a stochastic process with finite state space in continuous time. Different states reflect the severity of the disease. States are of two types: transient and absorbing. An absorbing state is a state that, once entered, cannot be left. Whereas, transient states are the ones with further transitions. The multi-state model is a useful tool to describe a subject's movements between different states in continuous time. The model computes transition probabilities for all possible transitions. ${ }^{11,12}$

We proposed a four-state continuous time homogeneous multi-state Markov model for the kidney disease progression data. A process is Markovian if the future depends only on the present. The model is visualized in Fig. 1.

The arrows indicate the possible transitions. The two-sided arrows imply reversible transitions. Transitions are reversible if the subject moves back and forth between two states. The three transient states (states with arrows out) considered are: state $1(K D I G O>60)$, state 2 $(30<K D I G O<60)$, and state $3(K D I G O<30)$. State 4 (death) is absorbing (state without arrows out). The process $(X(t), t \in T)$ with state space $S=\{1,2,3,4\}$ describes the state occupied at time $t$. The time variable is measured in days starting from hospital admission. The multistate model characterizes the transition probabilities. The transition probabilities (functions of time) are given by:

$P_{i j}(s, t)=P\left(X_{t}=j \mid X_{s}=i\right)$ for $i, j \in S, \cdot s \leq t$

Covariate effects (age, gender, hypertension, diabetes, etc.) on the bidirectional transition rates were estimated. The estimate of the hazard, $\lambda_{i j}$ is given by:

$\lambda_{i j}(t)=\lim _{\Delta t \rightarrow 0} \frac{P\left(X_{t+\Delta t}=j \mid X_{t}=i\right)}{\Delta t}$.

where $\lambda_{i j}$ is the instantaneous risk of moving from state $i$ to state $j$. The transition intensity matrix is given by: 
Table 3

Demographic and clinical characteristics.

\begin{tabular}{ll}
\hline Variables & $\mathrm{n}(\%)$ or mean (SD) \\
\hline Age in years (SD) & $54(17)$ \\
Gender - male (\%) & $166(73.78)$ \\
ICU - yes (\%) & $194(86.22)$ \\
Undergone surgery - yes (\%) & $119(52.89)$ \\
AKI - yes (\%) & $59(26.22)$ \\
Pneumonia - yes (\%) & $123(54.67)$ \\
CKD - yes (\%) & $30(13.33)$ \\
Sepsis - yes (\%) & $115(51.11)$ \\
Type 2 diabetes - yes (\%) & $78(34.67)$ \\
HTN - yes (\%) & $87(38.67)$ \\
\hline
\end{tabular}

Continuous variables are presented as mean (SD); categorical variables as frequency (\%).

$Q(t)=\left[\begin{array}{cccc}-\lambda_{1 .} & -\lambda_{12} & -\lambda_{13} & -\lambda_{14} \\ -\lambda_{21} & -\lambda_{2 .} & -\lambda_{23} & -\lambda_{24} \\ -\lambda_{31} & -\lambda_{32} & -\lambda_{3} & -\lambda_{34} \\ -\lambda_{41} & -\lambda_{42} & -\lambda_{43} & -\lambda_{4}\end{array}\right]$

$Q$ is the $n \times n$ transition intensity matrix, where, $n$ is the maximum number of states involved in the process. The transition intensity gives the instantaneous rate of transition from one state to another. The entry $(i, j)$ is 0 when there is no transition possible from $i$ to $j$. Diagonal entries are: $\lambda_{i i}(t)=\lambda_{i}=-\sum_{i \neq j} \lambda_{i j}(t)$ for all $i \in S$. State 4 being an absorbing state, the probability of moving out of this state is zero. The sum of the elements of each row of the transition matrix is zero. Fitting a multi-state model is the process of finding the unknown transition intensities that maximize the likelihood. ${ }^{13}$

The multi-state model estimates the mean sojourn time in each state, which is the average period a patient spends in a transient state in a single stay before moving to other states. The expected sojourn time is calculated as $-1 / \lambda_{j j}$, where $\lambda_{j j}$ is the $j^{\text {th }}$ diagonal entry of $Q(t)$.

Transition probabilities are computed from transition intensities as $P(t)=\exp [Q(t)]$. The transition probability matrix is given by:

$P(t)=\left[\begin{array}{llll}P_{11} & P_{12} & P_{13} & P_{14} \\ P_{21} & P_{22} & P_{23} & P_{24} \\ P_{31} & P_{32} & P_{33} & P_{34} \\ P_{41} & P_{42} & P_{43} & P_{44}\end{array}\right]$

The row sum of $P$ is equal to one. For the absorbing state $j, P_{j j}(s, t)=$ 1.

The likelihood function is formed with the transition probabilities. This likelihood function, $L(Q)$ is given by,

$L(Q)=\prod_{i, j} L_{i, j}=\prod_{i, j} P_{s\left(t_{i j}\right) s\left(t_{i, j+1}\right)}\left(t_{i, j+1}-t_{i j}\right)$

Where the element $L_{i, j}$ is the entry of the $s\left(t_{i j}\right)^{\text {th }}$ row and $s\left(t_{i, j+1}\right)^{\text {th }}$ column in the transition probability matrix.

The proportional hazards regression model was used to incorporate the covariate effects on transition intensities. Given a covariate vector $Z$,

$\lambda_{i j}[t \mid Z(t)]=\lambda_{i j 0} \quad \exp \left[\beta_{i j}^{T} Z(t)\right]$

$\beta_{i j}$ is the vector of regression coefficients.

We used the package $m s m$ in $\mathrm{R}$ version 4.0.2 to perform the multistate analysis. ${ }^{14}$ The model selection was done with the likelihood ratio test.

\section{Results}

\subsection{Exploratory data analysis}

This retrospective study aimed to model the kidney disease progression of patients receiving colistin during their hospital stay. Out of
Table 4

Summary statistics of length of stay.

\begin{tabular}{lllllll}
\hline Mean & SD & $\mathrm{Q}_{1}$ & Median & $\mathrm{Q}_{3}$ & Min & Max \\
\hline 25.30 & 18.50 & 14 & 21 & 30 & 4 & 164 \\
\hline
\end{tabular}

Table 5

Mean and median survival time.

\begin{tabular}{llllll}
\hline Mean & SE & $95 \%$ CI & Median & SE & $95 \%$ CI \\
\hline 82.39 & 7.38 & $(67.94,96.85)$ & 38 & 8.68 & $(20.98,55.02)$ \\
\hline
\end{tabular}

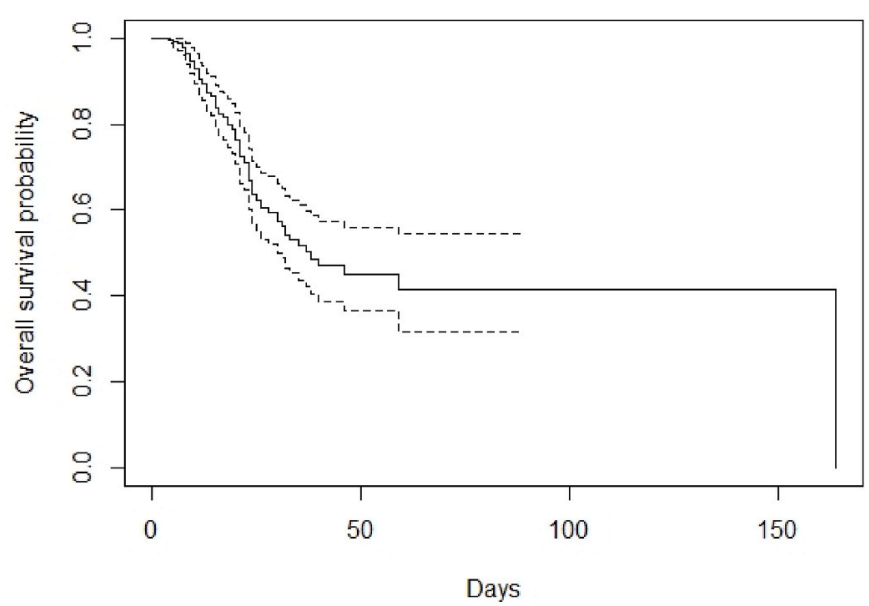

Fig. 2. Kaplan Meier estimates of the survival function.

Table 6

Summary of the transitions.

\begin{tabular}{llllll}
\hline From & & & To & \\
\cline { 3 - 6 } & $\mathbf{1}$ & & $\mathbf{2}$ & $\mathbf{3}$ & $\mathbf{4}$ \\
\hline 1 & 226 & 71 & 10 & 32 \\
2 & 47 & 28 & 50 & 27 \\
3 & 11 & 35 & 115 & 24 \\
\hline
\end{tabular}

the 225 patients, 83 (37\%) patients died in the hospital, and 142 (63\%) patients got discharged alive. Descriptive statistics can be found in Table 3 and Table 4. The median (IQR) length of stay was 21 (16) days. There were 166 male patients and 59 female patients admitted to the hospital. The median (IQR) age of the patients was 57 (26) and mean $( \pm S D)$ is $54( \pm 17)$. There were $87(39 \%)$ patients with hypertension, 78 (35\%) patients with diabetes, 115 (51\%) patients with sepsis, 30 (13.3\%) patients with Chronic Kidney Disease (CKD), 123 (55\%) patients with pneumonia, and 59 (26\%) patients had Acute Kidney Injury (AKI). Among the hospitalized patients, 194 (86\%) were admitted to ICU and 119 (53\%) patients had undergone a surgery.

Among the 225 patients, 83 (37\%) patients died in the hospital and the remaining 142 (63\%) patients survived being alive. The median survival time was 38 days (Table 5). Fig. 2 represents the Kaplan Meier estimates of the survival function.

\subsection{Multi-state analysis}

There were 126 patients in state 1,48 patients in state 2 , and 51 patients in state 3 at the time of admission. The transition matrix of the process is given in Table 6. Since the transitions were reversible, there was more than one transition of the same type in the same patient.

There were 32 transitions from state 1 to state 4 , indicating 32 
Table 7

Estimates of mean sojourn time.

\begin{tabular}{llll}
\hline States & Estimate & SE & $95 \%$ CI \\
\hline State 1 & 4.48 & 0.84 & $(3.10,6.48)$ \\
State 2 & 0.69 & 0.22 & $(0.37,1.29)$ \\
State 3 & 1.40 & 0.69 & $(0.53,3.68)$ \\
\hline
\end{tabular}

Table 8

Hazard ratios and 95\% confidence intervals for demographic variables.

\begin{tabular}{lll}
\hline Transitions & Gender & $\log ($ age $)$ \\
\hline $1 \rightarrow 2$ & $\mathbf{2 . 5 5 ( 1 . 3 1 , 4 . 9 2 )}$ & $3.26(0.97,10.99)$ \\
$1 \rightarrow 3$ & $0.85(0.25,1.65)$ & $2.05(0.50,5.62)$ \\
$1 \rightarrow 4$ & $0.67(0.18,2.48)$ & $0.29(0.06,1.31)$ \\
$2 \rightarrow 1$ & $\mathbf{4 . 6 6 ( 1 . 9 7 , 1 0 . 9 8 )}$ & $0.90(0.25,3.24)$ \\
$2 \rightarrow 3$ & $\mathbf{0 . 0 2}(\mathbf{0 . 0 0 , 0 . 4 0 )}$ & $1.85(0.42,8.17)$ \\
$2 \rightarrow 4$ & $0.85(0.34,2.11)$ & $0.98(0.18,5.32)$ \\
$3 \rightarrow 1$ & $1.01(0.53,2.01)$ & $1.68(0.03,3.98)$ \\
$3 \rightarrow 2$ & $\mathbf{0 . 0 2}(\mathbf{0 . 0 0 , 0 . 3 0 )}$ & $4.84(1.00,21.75)$ \\
$3 \rightarrow 4$ & $\mathbf{0 . 2 4}(\mathbf{0 . 0 7 , 0 . 8 9 )}$ & $1.40(0.20,9.76)$ \\
\hline
\end{tabular}

Bold face values indicate significance.

patients who belonged to state 1 died in the hospital. Similarly, there were 27 and 24 deaths from state 2 and state 3 respectively. The diagonal entries indicate the number of instances when the patients remained in the same state in subsequent time points. The estimated mean sojourn time is given in Table 7.

msm package was used to perform the multi-state analysis to obtain the effects of covariates on the transition intensities. Univariate multistate models were built with clinically significant covariates. Covariates that showed statistical significance (p-value $<0.05$ ) in the univariate analysis were considered in the final model. Hazard ratios $(95 \%$ confidence intervals) of each transition are shown in Table 8 and Table 9.

Male patients in state 1 are at high risk [HR: 2.55; 95\% CI (1.31-4.92)] of disease progression compared to females. However, the chance of regression is also more for male patients in state 2. Compared to the females in state 3 , males have an $86 \%$ less chance of an adverse outcome. The presence of diabetes showed a mixed effect in different transitions. Hypertensive patients in state 2 are at more risk of disease progression compared to the others and their chance of regression from state 3 is also less. Sepsis was a risk factor and showed a significant effect in the transition from state 1 and state 2 to the absorbing state. This indicates that patients with sepsis are at high risk of death. Similarly, patients who have undergone surgery are at more risk of death in state 3 . Due to the imbalance in some groups, there may be some spurious associations not indicating clinical significance.

\section{Discussion and conclusion}

Kidney disease is an important public health problem. However, early interventions can avoid kidney problems permanently. Modelling the disease progression helps in understanding the expected burden of the disease which can be further useful for the national public health policymakers.

Some of the non-fatal intermediate events give more insight into disease progression. In most of the studies, these events are often ignored. However, these intermediate events are of considerable importance for clinicians to improve treatment plans. The multi-state Markov model has been used to understand the progression of several chronic diseases involving transitions across different intermediate states indicating the severity of the disease in continuous time. The model provides more insight into the complex event pattern and thus it can be used as an effective tool to study the effectiveness of treatments. The multi-state model is being used in cancer studies as there are different stages of cancer that constitute different transitions. ${ }^{4,5}$ Similarly, there are studies conducted to identify the risk factors influencing AIDS progression in HIV patients. ${ }^{6-8}$ Diseases such as diabetes, chronic kidney disease, etc. can also be efficiently handled with a multi-state model rather than a simple survival model. ${ }^{9,10}$ However, less number of applications are found in the field of chronic kidney disease progression. The most recent study that used a multi-state model to understand the chronic kidney disease progression was by Grover et al. ${ }^{10}$

We proposed a 4 state multi-state model for the kidney disease progression of patients receiving colistin. An attempt was made to provide a simple demonstration of the multi-state model targeting health professionals. With the availability of advanced software tools, the analysis is simple and effective. Even though we emphasized on general Markov model, there are different types of multi-state models according to the nature of the process. ${ }^{1,2,15-18}$

The following basic steps are recommended to use the multi-state model: (i) define the process by identifying different states and possible transitions as shown in Fig. 1 and Table 2. Keep the number of states minimal for smooth functioning of the model (ii) document the data properly as shown in Table 1. (iii) use appropriate software and package to fit the model (iv) interpret the results without losing the advantages of multi-state model over the other standard survival analysis techniques.

The median length of stay was 21 days and the median survival time was 38 days. The results indicate that gender, diabetes, hypertension, sepsis, and surgery are significant factors affecting the progression or regression of kidney disease. The results obtained from the present study are limited in power due to the smaller number of subjects. Therefore, these findings need to be generalized with caution. Subsequently, different stages of kidney disease were merged due to the smaller sample size. This would affect the generalizability of the findings as the exact picture of the transition patterns of kidney disease progression is not included. $^{8}$

Table 9

Hazard ratios and 95\% confidence intervals for comorbidities and procedures.

\begin{tabular}{|c|c|c|c|c|c|}
\hline Transitions & Diabetes & HTN & Sepsis & Pneumonia & Surgery \\
\hline $1 \rightarrow 2$ & $0.39(0.17,0.92)$ & $1.78(0.80,3.99)$ & $2.73(1.25,5.92)$ & $0.78(0.40,1.53)$ & $1.57(0.78,3.13)$ \\
\hline $1 \rightarrow 3$ & $0.63(0.10,1.02)$ & $1.25(0.53,2.89)$ & $0.05(0.02,7.35)$ & $0.55(0.17,1.23)$ & $2.31(0.91,5.21)$ \\
\hline $1 \rightarrow 4$ & $1.07(0.24,4.72)$ & $2.48(0.62,9.88)$ & $10.23(3.09,33.86)$ & $1.13(0.36,3.48)$ & $2.03(0.10,5.67)$ \\
\hline $2 \rightarrow 1$ & $0.33(0.13,0.82)$ & $1.23(0.51,2.97)$ & $1.02(0.44,2.31)$ & $0.64(0.30,1.37)$ & $1.80(0.82,3.95)$ \\
\hline $2 \rightarrow 3$ & $17.11(4.52,64.73)$ & $1.66(1.09,2.53)$ & $1.89(0.73,4.85)$ & $0.46(0.17,1.23)$ & $1.63(0.67,3.93)$ \\
\hline $2 \rightarrow 4$ & $1.51(0.56,4.04)$ & $2.18(0.86,5.51)$ & $5.72(1.73,18.90)$ & $1.03(0.45,2.34)$ & $2.29(0.80,6.46)$ \\
\hline $3 \rightarrow 1$ & $2.08(0.76,3.58)$ & $0.88(0.31,1.51)$ & $3.12(0.91,6.21)$ & $0.85(0.76,1.21)$ & $4.1(0.23,10.02)$ \\
\hline $3 \rightarrow 2$ & $9.44(2.67,33.40)$ & $0.07(0.02,0.27)$ & $3.07(0.13,8.33)$ & $0.92(0.33,2.55)$ & $1.68(0.68,4.17)$ \\
\hline $3 \rightarrow 4$ & $0.91(0.31,2.57)$ & $0.07(0.01,2.39)$ & $4.71(0.89,25.06)$ & $0.84(0.21,3.18)$ & $3.41(2.23,4.11)$ \\
\hline
\end{tabular}

Bold face values indicate significance; HTN: Hypertension. 
The $m s m$ package in $\mathrm{R}$ version 4.0.2 was used to fit the multi-state model. As the limitations listed in the documentation of the $m s m$ package ${ }^{14}$ inclusion of more number of covariates was challenging due to the convergence issues. Therefore, for more complex problems, mstate package can be used.

The findings from a multi-state model will be useful for public health policymakers to implement the policies and treatment plans to improve the survival of the patients. Moreover, modeling the disease progression helps in understanding the expected burden of the disease.

\section{Ethics approval and consent to participate}

The study presents a secondary analysis. The ethical approval was obtained for the clinical companion paper.

\section{Funding}

This research did not receive any funding.

\section{Availability of data and materials}

The datasets used and/or analyzed during the current study are available from the corresponding author on reasonable request.

\section{Declaration of competing interest}

The authors have no conflicts of interest to disclose.

\section{References}

1 Andersen PK. Multistate models in survival analysis: a study of nephropathy and mortality in diabetes. Stat Med. 1988;7(6):661-670.
2 Andersen PK, Esbjerg S, Sørensen TI. Multi-state models for bleeding episodes and mortality in liver cirrhosis. Stat Med. 2000;19(4):587-599.

3 Amorim LD, Cai J. Modelling recurrent events: a tutorial for analysis in epidemiology. Int J Epidemiol. 2015;44(1):324-333.

4 Le-Rademacher JG, Peterson RA, Therneau TM, et al. Application of multi-state models in cancer clinical trials. Clin Trials. 2018;15(5):489-498.

5 Putter H, van der Hage J, de Bock GH, et al. Estimation and prediction in a multistate model for breast cancer. Biom J. 2006;48(3):366-380.

6 Hamidi O, Tapak L, Poorolajal J, et al. Identifying risk factors for progression to AIDS and mortality post-HIV infection using illness-death multistate model. Clin Epidemiology Glob Health. 2017;5(4):163-168.

7 Tapak L, Kosorok MR, Sadeghifar M, et al. Multistate recursively imputed survival trees for time-to-event data analysis: an application to AIDS and mortality post-HIV infection data. BMC Med Res Methodol. 2018;18(1):1-2.

8 Matsena Zingoni Z, Chirwa TF, Todd J, et al. HIV disease progression among antiretroviral therapy patients in Zimbabwe: a multistate Markov model. Front Public Health. 2019;7:326.

9 Aliyari R, Hajizadeh E, Aminorroaya A, et al. Multistate models to predict development of late complications of type 2 diabetes in an open cohort study. Diabetes Metab Syndr Obes. 2020;13:1863.

10 Grover G, Sabharwal A, Kumar S, et al. A multi-state markov model for the progression of chronic kidney disease. Turkiye Klinikleri J Biostat. 2019;11(1):1-4.

11 Keiding N, Klein JP, Horowitz MM. Multi-state models and outcome prediction in bone marrow transplantation. Stat Med. 2001;20(12):1871-1885.

12 Klein JP, Qian C. Modeling multistate survival illustrated in bone marrow transplantation. Biometrics. 1996:93-102.

13 Manzini G, Ettrich TJ, Kremer M, et al. Advantages of a multi-state approach in surgical research: how intermediate events and risk factor profile affect the prognosis of a patient with locally advanced rectal cancer. BMC Med Res Methodol. 2018;18(1); $1-11$.

14 Jackson CH. Multi-state models for panel data: the msm package for R. J Stat Software. 2011;38(8):1-29.

15 Marshall G, Jones RH. Multi-state models and diabetic retinopathy. Stat Med. 1995 Sep 30;14(18):1975-1983.

16 Meira-Machado L, de Uña-Álvarez J, Cadarso-Suárez C, et al. Multi-state models for the analysis of time-to-event data. Stat Methods Med Res. 2009;18(2):195-222.

17 Begun A, Icks A, Waldeyer R, et al. Identification of a multistate continuous-time nonhomogeneous Markov chain model for patients with decreased renal function. Med Decis Making. 2013 Feb;33(2):298-306.

18 O'Keeffe AG, Su L, Farewell VT. Correlated multistate models for multiple processes: an application to renal disease progression in systemic lupus erythematosus. Royal Statistical Society. 2018;67(4):841-860. 